The Journal of $\mathbf{N}_{\text {onlinear }} \mathbf{S}$ ciences and Applications

http://www.tjnsa.com

\title{
SOLVABILITY OF NONLINEAR HAMMERSTEIN QUADRATIC INTEGRAL EQUATIONS
}

\author{
A. M. A EL-SAYED ${ }^{1}$ AND H. H. G HASHEM ${ }^{2 *}$ \\ This paper is dedicated to Professor Hemant Kumar Nashine
}

\begin{abstract}
We are concerning with a nonlinear Hammerstein quadratic integral equation. We prove the existence of at least one positive solution $x \in L_{1}$ under Carathèodory condition. Secondly we will make a link between Peano condition and Carathèodory condition to prove the existence of at least one positive continuous solution. Finally the existence of the maximal and minimal solutions will be proved.
\end{abstract}

\section{INTRODUCTION AND PRELIMINARIES}

Quadratic integral equations are often applicable in the theory of radiative transfer, kinetic theory of gases, in the theory of neutron transport and in the traffic theory. The quadratic integral equation can be very often encountered in many applications (see[1]-4] and [8]-[11]).

Let $I=[0,1], L_{1}=L_{1}[0,1]$ be the space of Lebesgue integrable functions on $I$ and $C=C[0,1]$ be the space of continuous functions defined on $I$.

Recently, the existence of a solution $x \in L_{1}$ for the nonlinear quadratic integral equation

$$
x(t)=a(t)+g(t, x(t)) \int_{0}^{t} k(t, s) f(s, x(s) d s, t \in[0,1]
$$

was studied in [9] by using Lusin and Dragoni theorems and applying SchauderTychonoff fixed point Theorem.

Date: Received: 13 March 2009; Revised: 20 April 2009.

* Corresponding author.

2000 Mathematics Subject Classification. Primary 39B82; Secondary 44B20, 46C05.

Key words and phrases. Hammerstein quadratic integral equation; Positive integrable solutions; Continuous solutions; Maximal and minimal solutions. 
Here we are concerning with the nonlinear Hammerstein quadratic integral equation

$$
x(t)=a(t)+g(t, x(t)) \int_{0}^{1} k(t, s) f(s, x(s)) d s t \in[0,1]
$$

by using the same assumptions assumed in [9]. Firstly, the existence of at least one $L_{1}$-positive solution of the nonlinear quadratic integral equation (1.1) will be proved where the functions $f$ and $g$ satisfy Carathèodory condition.

Secondly, the existence of at least one positive continuous solution for the quadratic integral equation (1.1) will be proved where $g$ is continuous and $f$ satisfies Carathèodory condition.

The following theorems will be needed in our investigations (see [6],[7] and [14]).

Theorem 1.1. Tychonov's fixed-point Theorem. Suppose $B$ is a complete, locally convex linear space and $S$ is a closed convex subset of $B$. Let the mapping $T: B \rightarrow B$ be continuous and $T(S) \subset S$. If the closure of $T(S)$ is compact, then $T$ has a fixed-point in $S$.

Theorem 1.2. Shauder fixed-point Theorem. Let $S$ be a convex subset of a Banach space $B$, let the mapping $T: S \rightarrow S$ be compact, continuous. Then $T$ has at least one fixed-point in $S$.

Theorem 1.3. Arzela-Ascoli Theorem. Let $E$ be a compact metric space and $C(E)$ the Banach space of real or complex valued continuous functions normed by

$$
\|f(t)\|=\max _{t \in E}|f(t)| .
$$

If $A=\left\{f_{n}\right\}$ is a sequence in $C(E)$ such that $f_{n}$ is uniformly bounded and equi-continuous. Then the closure of $A$ is compact.

Theorem 1.4. Lusin Theorem. Let $m:[0,1] \rightarrow R$ be a measurable function. For any $\epsilon>0$ there exists a closed subset $A_{\epsilon}$ of $[0,1]$, meas. $\left(A_{\epsilon}^{c}\right)<\epsilon$, such that $m$ restricted to $A_{\epsilon}$ is continuous.

Theorem 1.5. Scorza Dragoni Theorem. Let $k:[0,1] \times[0,1] \rightarrow R$ be a function satisfying Carathèodory condition (i.e. measurable in $t$ for all $s \in[0,1]$ and continuous in $s$ for all $t \in[0,1])$. For any $\epsilon>0$ there exists a closed subset $A_{\epsilon}$ of $[0,1]$, meas. $\left(A_{\epsilon}^{c}\right)<\epsilon$, such that $k$ restricted to $A_{\epsilon} \times[0,1]$ is continuous.

\section{2. $L_{1}$-POSITIVE SOLUTION}

Let $I=[0,1]$, and consider the assumptions:

(i) $a: I \rightarrow R_{+}=[0,+\infty)$ is integrable on $I$;

(ii) $f, g: I \times R_{+} \rightarrow R_{+}$satisfy Carathèodory condition (i.e. measurable in $t$ for all $x \in R_{+}$and continuous in $x$ for all $\left.t \in[0,1]\right)$ and there exist two functions $m_{1}, m_{2} \in L_{1}$ such that

$$
g(t, x) \leq m_{1}(t), f(t, x) \leq m_{2}(t) \forall(t, x) \in I \times R_{+} ;
$$


(iii) $k:[0,1] \times[0,1] \rightarrow R$ satisfies Carathèodory condition (i.e. measurable in $t$ for all $s \in[0,1]$ and continuous in $s$ for all $t \in[0,1])$.

For the existence of at least one $L_{1}$-positive solution of the nonlinear quadratic integral equation (1.1) we have the following theorem.

Theorem 2.1. Let the assumptions (i)-(iii) be satisfied. Then the nonlinear quadratic integral equation (1.1) has at least one $L_{1}$-positive solution $x$.

Proof. Consider the set $Q \subset L_{1}$ such that

$$
Q=\left\{x \in L_{1},|x(t)| \leq x_{0}(t) \text { a.e. }\right\}
$$

where

$$
x_{0}(t)=a(t)+m_{1}(t) \int_{0}^{1} k(t, s) m_{2}(s) d s .
$$

The set $Q$ can be shown to be nonempty, bounded, closed and convex in $L_{1}$. Let $H$ be the operator defined by

$$
(H x)(t)=a(t)+g(t, x(t)) \int_{0}^{1} k(t, s) f(s, x(s)) d s, t \in I .
$$

We shall prove that $H: Q \rightarrow Q$. For that let $x \in Q$, then

$$
|(H x)(t)| \leq|a(t)|+m_{1}(t) \int_{0}^{1} k(t, s) m_{2}(s) d s=x_{0}(t),
$$

so $H x \in Q$ and hence $H Q \subset Q$.

To apply Schauder fixed-point Theorem, we shall prove that $H Q$ is relatively compact in $L_{1}$.

By using Lusin and Scorza Dragoni Theorems, we can find a closed subset $A_{n}$ of $[0,1]$, with meas. $\left(A_{n}^{c}\right)<\frac{1}{n}$ such that $a(t), \quad m_{1}(t),\left.\quad k\right|_{A_{n} \times[0,1]}$ and $\left.g\right|_{A_{n} \times Q}$ are uniformly continuous on $A_{n}$.

Assume that $x_{h}$ is any sequence in $Q$, then for $t_{1}, t_{2} \in A_{n}$, we have

$$
\begin{aligned}
& \left(H x_{h}\right)\left(t_{2}\right)-\left(H x_{h}\right)\left(t_{1}\right)=a\left(t_{2}\right)-a\left(t_{1}\right) \\
& +g\left(t_{2}, x_{h}\left(t_{2}\right)\right) \int_{0}^{1} k\left(t_{2}, s\right) f\left(s, x_{h}(s)\right) d s-g\left(t_{1}, x_{h}\left(t_{1}\right)\right) \int_{0}^{1} k\left(t_{1}, s\right) f\left(s, x_{h}(s)\right) d s \\
& =a\left(t_{2}\right)-a\left(t_{1}\right)+g\left(t_{2}, x_{h}\left(t_{2}\right)\right) \int_{0}^{1} k\left(t_{2}, s\right) f\left(s, x_{h}(s)\right) d s \\
& -g\left(t_{1}, x_{h}\left(t_{1}\right)\right) \int_{0}^{1} k\left(t_{1}, s\right) f\left(s, x_{h}(s)\right) d s+g\left(t_{1}, x_{h}\left(t_{1}\right)\right) \int_{0}^{1} k\left(t_{2}, s\right) f\left(s, x_{h}(s)\right) d s \\
& \quad-g\left(t_{1}, x_{h}\left(t_{1}\right)\right) \int_{0}^{1} k\left(t_{2}, s\right) f\left(s, x_{h}(s)\right) d s \\
& \leq a\left(t_{2}\right)-a\left(t_{1}\right)+\left[g\left(t_{2}, x_{h}\left(t_{2}\right)\right)-g\left(t_{1}, x_{h}\left(t_{1}\right)\right)\right] \int_{0}^{1} k\left(t_{2}, s\right) f\left(s, x_{h}(s)\right) d s \\
& +g\left(t_{1}, x_{h}\left(t_{1}\right)\right) \int_{0}^{1}\left\{k\left(t_{2}, s\right)-k\left(t_{1}, s\right)\right\} f\left(s, x_{h}(s)\right) d s
\end{aligned}
$$


Then we get

$$
\begin{aligned}
\mid\left(H x_{h}\right)\left(t_{2}\right)- & \left(H x_{h}\right)\left(t_{1}\right)|\leq| a\left(t_{2}\right)-a\left(t_{1}\right)\left|+m_{1}\left(t_{1}\right) \int_{0}^{1}\right| k\left(t_{2}, s\right)-k\left(t_{1}, s\right) \mid m_{2}(s) d s \\
& +\left|g\left(t_{2}, x_{h}\left(t_{2}\right)\right)-g\left(t_{1}, x_{h}\left(t_{1}\right)\right)\right| \int_{0}^{1} k\left(t_{2}, s\right) m_{2}(s) d s
\end{aligned}
$$

This means that the sequence $\left\{H x_{h}\right\}$ is sequence of equi-continuous functions on $A_{n}$ and we can prove that this sequence is uniformly bounded.

Now

$$
\begin{aligned}
\left|\left(H x_{h}\right)(t)\right| & =|a(t)|+m_{1}(t) \int_{0}^{1} k(t, s) m_{2}(s) d s \\
\leq & M_{1}+M_{2} K \int_{0}^{1} m_{2}(s) d s
\end{aligned}
$$

where $|a(t)|_{A_{n}} \leq M_{1}, \quad\left|m_{1}(t)\right|_{A_{n}} \leq M_{2}$ and $\left.k\right|_{A_{n} \times[0,1]} \leq K$.

Hence by Arzela-Ascoli Theorem $H x_{h}$ is relatively compact subset of $C\left(A_{n}\right)$ and this can be done for each $n \in N$. this implies the existence of convergent subsequence $\left\{x_{h_{j}}\right\}$ of $\left\{x_{h}\right\}$ in each $C\left(A_{n}\right)$. Given $\epsilon>0$ and choose $n_{1} \in N$ so that meas $\left(A_{n_{1}}\right)<\epsilon$, then

$$
\begin{aligned}
\int_{0}^{T} \mid H x_{h_{j}} & -H x_{h_{l}}\left|d t=\int_{A_{n_{1}}^{c}}\right| H x_{h_{j}}-H x_{h_{l}} \mid d t \\
& +\int_{A_{n_{1}}}\left|H x_{h_{j}}-H x_{h_{l}}\right| d t .
\end{aligned}
$$

Since $C\left(A_{n}\right)$ is complete metric space, hence this subsequence is a Cauchy sequence in each $C\left(A_{n}\right), n=1,2,3, \ldots$

That is for given $\epsilon>0$ and $j, l$ are arbitrary large we have

$$
\left\|H x_{h_{j}}-H x_{h_{l}}\right\|_{C\left(A_{n}\right)}<\epsilon \text {. }
$$

But we want to prove that the set $H Q$ is relatively compact in $L_{1}$, that is $\overline{H Q}$ is compact in $L_{1}$.

To do this, we will prove that the sequence $\left\{H x_{h}\right\}$ is convergent in $L_{1}$, since $L_{1}$ is complete metric space, then it is sufficient to prove that the subsequence $\left\{H x_{h_{j}}\right\}$ is a Cauchy sequence in $L_{1}$.

i.e. $\forall \eta>0, \exists N(\eta)$ and $\int_{A_{n}} x_{0}(t) d t<\eta / 4$ such that

$$
\left\|H x_{h_{j}}-H x_{h_{l}}\right\|_{L_{1}}<\eta, j, l>N(\eta) .
$$

Now from (2.1) and (2.2) we have

$$
\begin{gathered}
\int_{0}^{T}\left|H x_{h_{j}}-H x_{h_{l}}\right| d t=\int_{A_{n}^{c}}\left|H x_{h_{j}}-H x_{h_{l}}\right| d t \\
+\int_{A_{n}}\left|H x_{h_{j}}-H x_{h_{l}}\right| d t . \\
\leq \int_{A_{n}^{c}}\left\{\left|H x_{h_{j}}\right|+\left|H x_{h_{l}}\right|\right\} d t+\left\|H x_{h_{j}}-H x_{h_{l}}\right\|_{C\left(A_{n}\right)}
\end{gathered}
$$




$$
\leq \eta / 4+\eta / 4+\left\|H x_{h_{j}}-H x_{h_{l}}\right\|_{C\left(A_{n}\right)} .
$$

Choose $N$ such that $l, j>N$, then (2.3) implies that $\left\|H x_{h_{j}}-H x_{h_{l}}\right\|_{C\left(A_{n}\right)} \leq \eta / 2$. This means that the subsequence $\left\{H x_{h_{j}}\right\}$ is a Cauchy sequence in $L_{1}$ which implies that $H Q$ is relatively compact in $L_{1}$. Then $H$ has at least one fixed point. Hence there exists at least one solution $x \in L_{1}$ of (1.1).

Since all conditions of Shauder's fixed-point Theorem hold, then $H$ has a fixed point in $Q$.

\section{Continuous solutions}

Let $I=[0,1]$, and consider the assumptions:

(i) $a: I \rightarrow R_{+}=[0,+\infty)$ is continuous on $I$;

(ii) $f: I \times R_{+} \rightarrow R_{+}$satisfies Carathéodory condition (i.e. measurable in $t$ for all $x \in R_{+}$and continuous in $x$ for all $\left.t \in[0,1]\right)$ and there exists function $m \in L_{1}$ such that

$$
f(t, x(t)) \leq m(t) \forall(t, x) \in I \times R_{+} ;
$$

(iii) $g: I \times R_{+} \rightarrow R_{+}$is continuous in $t, x$ and $|g(t, x)| \leq M$;

(iv) $k:[0,1] \times[0,1] \rightarrow R$ satisfies Carathèodory condition (i.e. measurable in $t$ for all $s \in[0,1]$ and continuous in $s$ for all $t \in[0,1])$.

Now for the existence of at least one positive continuous solution of the nonlinear quadratic integral equation (1.1) we have the following theorem.

Theorem 3.1. Let the assumptions (i)-(iv) be satisfied. Then the nonlinear quadratic integral equation (1.1) has at least one positive solution $x \in C(I)$.

Proof. We shall use Tychonov's fixed point Theorem to prove this theorem It can be verified that [7] $C$ is complete locally convex linear space. Define a subset $S$ of $C$ by

$$
S=\left\{x \in C:|x(t)| \leq M_{2}\right\}, t \in[0,1],
$$

where $M_{2}$ is a positive constant. It is clear that the set $S$ is closed and convex. Let $H$ be an operator defined by

$$
(H x)(t)=a(t)+g(t, x(t)) \int_{0}^{1} k(t, s) f(s, x(s)) d s, \forall x \in S .
$$

Assumptions (ii) and (iii) imply that $H: S \rightarrow C$ is continuous operator in $x$. We shall prove that $H S \subset S$.

For every $x \in S$ we have

$$
\begin{aligned}
|(H x)(t)| & \leq|a(t)|+M \int_{0}^{1} k(t, s) m(s) d s t \in[0,1] \\
& \leq M_{1}+M \int_{0}^{1} k(t, s) m(s) d s=M_{2},
\end{aligned}
$$


SOLVABILITY OF NONLINEAR HAMMERSTEIN QUADRATIC INTEGRAL EQUATION\$57

where $|a(t)| \leq M_{1}$. Then, $H x \in S$ and hence $H S \subset S$. Also for $t_{1}$ and $t_{2} \in[0,1]$ we can have

$$
\begin{gathered}
(H x)\left(t_{2}\right)-(H x)\left(t_{1}\right)=a\left(t_{2}\right)-a\left(t_{1}\right)+g\left(t_{2}, x\left(t_{2}\right)\right) \int_{0}^{1} k\left(t_{2}, s\right) f(s, x(s)) d s \\
-g\left(t_{1}, x\left(t_{1}\right)\right) \int_{0}^{1} k\left(t_{1}, s\right) f(s, x(s)) d s+g\left(t_{1}, x\left(t_{1}\right)\right) \int_{0}^{1} k\left(t_{2}, s\right) f(s, x(s)) d s \\
\quad-g\left(t_{1}, x\left(t_{1}\right)\right) \int_{0}^{1} k\left(t_{2}, s\right) f(s, x(s)) d s \\
=a\left(t_{2}\right)-a\left(t_{1}\right)+\left[g\left(t_{2}, x\left(t_{2}\right)\right)-g\left(t_{1}, x\left(t_{1}\right)\right)\right] \int_{0}^{1} k\left(t_{2}, s\right) f(s, x(s)) d s \\
+g\left(t_{1}, x\left(t_{1}\right)\right) \int_{0}^{1}\left[k\left(t_{2}, s\right)-k\left(t_{1}, s\right)\right] f(s, x(s)) d s
\end{gathered}
$$

Using assumptions (ii) (iii) then, we have

$$
\begin{gathered}
\left|(H x)\left(t_{2}\right)-(H x)\left(t_{1}\right)\right| \leq\left|a\left(t_{2}\right)-a\left(t_{1}\right)\right|+M \int_{0}^{1}\left|k\left(t_{2}, s\right)-k\left(t_{1}, s\right)\right| m(s) d s \\
+\left|g\left(t_{2}, x\left(t_{2}\right)\right)-g\left(t_{1}, x\left(t_{1}\right)\right)\right| \int_{0}^{1} k\left(t_{2}, s\right) m(s) d s
\end{gathered}
$$

This means that the functions of $H S$ are equi-continuous on $[0,1]$, then by Arzela-Ascoli Theorem the closure of $H S$ is compact.

Hence, all conditions of Tychonov fixed-point Theorem hold, then $H$ has a fixed point in $S$.

\section{Maximal and minimal solutions}

Definition 4.1. 13 Let $q(t)$ be a solution of the nonlinear Hammerstein quadratic integral equation (1.1). Then $q(t)$ is said to be a maximal solution of (1.1) if every solution $x(t)$ of (1.1) satisfies the inequality $x(t)<q(t)$. A minimal solution $s(t)$ can be defined by similar way by reversing the above inequality i.e. $x(t)>s(t)$.

We shall use the following lemma to prove the existence of the maximal and minimal solutions.

Lemma 4.2. Let $a(t)$ is continuous function on $I$ and $k(t, s)$ satisfies the assumption (iv) of Theorem 3.1. Let $f(t, x), g(t, x) \in L_{1}$ and $x(t), y(t)$ are continuous functions on $[0,1]$ satisfying

$$
\begin{aligned}
& x(t) \leq a(t)+g(t, x(t)) \int_{0}^{1} k(t, s) f(s, x(s)) d s, \\
& y(t) \geq a(t)+g(t, y(t)) \int_{0}^{1} k(t, s) f(s, y(s)) d s
\end{aligned}
$$

and one of them is strict. If $f(t, x), g(t, x)$ are monotonic nondecreasing in $x$, then

$$
x(t)<y(t), t>0 .
$$


Proof. Let the conclusion (4.1) be false, then there exists $t_{1}$ such that

$$
x\left(t_{1}\right)=y\left(t_{1}\right), t_{1}>0
$$

and

$$
x(t)<y(t), 0<t<t_{1} .
$$

From the monotonicity of $f(t, x), g(t, x)$ in $x$, we get

$$
\begin{aligned}
x\left(t_{1}\right) & \leq a\left(t_{1}\right)+g\left(t_{1}, x\left(t_{1}\right)\right) \int_{0}^{1} k\left(t_{1}, s\right) f(s, x(s)) d s, \\
& <a\left(t_{1}\right)+g\left(t_{1}, y\left(t_{1}\right)\right) \int_{0}^{1} k\left(t_{1}, s\right) f(s, y(s)) d s \\
& <y\left(t_{1}\right),
\end{aligned}
$$

which contradicts the fact that $x\left(t_{1}\right)=y\left(t_{1}\right)$, then $x(t)<y(t)$.

Theorem 4.3. Let the assumptions of Theorem 3.1 be satisfied and if $f(t, x), g(t, x)$ are nondecreasing in $x$ on $I$. Then there exist maximal and minimal solutions of the nonlinear quadratic integral equation (1.1).

Proof. Firstly we shall prove the existence of the maximal solution of (1.1). Let $\epsilon>0$ be given. Now consider the quadratic integral equation

$$
x_{\epsilon}(t)=a(t)+g_{\epsilon}\left(t, x_{\epsilon}(t)\right) \int_{0}^{1} k(t, s) f_{\epsilon}\left(s, x_{\epsilon}(s)\right) d s t \in[0,1],
$$

where

$$
\begin{aligned}
& f_{\epsilon}\left(t, x_{\epsilon}(t)\right)=f\left(t, x_{\epsilon}(t)\right)+\epsilon, \\
& g_{\epsilon}\left(t, x_{\epsilon}(t)\right)=g\left(t, x_{\epsilon}(t)\right)+\epsilon,
\end{aligned}
$$

Clearly the functions $f_{\epsilon}\left(t, x_{\epsilon}\right)$ and $g_{\epsilon}\left(t, x_{\epsilon}\right)$ satisfy assumptions (ii),(iii) of theorem 3.1 and therefore equation (4.2) at least a positive solution $x_{\epsilon}(t) \in C(I)$. Let $\epsilon_{1}$ and $\epsilon_{2}$ be such that $0<\epsilon_{2}<\epsilon_{1}<\epsilon$. Then

$$
\begin{gathered}
x_{\epsilon_{2}}(t)=a(t)+g_{\epsilon_{2}}\left(t, x_{\epsilon_{2}}(t)\right) \int_{0}^{1} k(t, s) f_{\epsilon_{2}}\left(s, x_{\epsilon_{2}}(s)\right) d s \\
x_{\epsilon_{2}}(t)=a(t)+\left(g\left(t, x_{\epsilon_{2}}(t)\right)+\epsilon_{2}\right) \int_{0}^{1} k(t, s)\left(f\left(s, x_{\epsilon_{2}}(s)\right)+\epsilon_{2}\right) d s \\
x_{\epsilon_{1}}(t)=a(t)+\left(g\left(t, x_{\epsilon_{1}}(t)\right)+\epsilon_{1}\right) \int_{0}^{1} k(t, s)\left(f\left(s, x_{\epsilon_{1}}(s)\right)+\epsilon_{1}\right) d s \\
x_{\epsilon_{1}}(t)>a(t)+\left(g\left(t, x_{\epsilon_{1}}(t)\right)+\epsilon_{2}\right) \int_{0}^{1} k(t, s)\left(f\left(s, x_{\epsilon_{1}}(s)\right)+\epsilon_{2}\right) d s .
\end{gathered}
$$

Applying Lemma 4.2 on (4.3) and (4.4), we have

$$
x_{\epsilon_{2}}(t)<x_{\epsilon_{1}}(t) \text { for } t \in I .
$$

As shown before the family of functions $x_{\epsilon}(t)$ is equi-continuous and uniformly bounded.

Hence by Arzela-Ascoli Theorem, there exists a decreasing sequence $\epsilon_{n}$ such that $\epsilon \rightarrow 0$ as $n \rightarrow \infty$ and $\lim _{n \rightarrow \infty} x_{\epsilon_{n}}(t)$ exists uniformly in $I$ and 
denote this limit by $q(t)$. From the continuity of the functions $f_{\epsilon}\left(t, x_{\epsilon}\right)$ and $g_{\epsilon}\left(t, x_{\epsilon}\right)$ in the second argument, we get

$$
q(t)=\lim _{n \rightarrow \infty} x_{\epsilon_{n}}(t)=a(t)+g(t, q(t)) \int_{0}^{1} k(t, s) f(s, q(s)) d s
$$

implies $q(t)$ as a solution of (1.1).

Finally, we shall show that $q(t)$ is the maximal solution of (1.1). To do this, let $x(t)$ be any solution of (1.1). Then

$$
\begin{gathered}
x_{\epsilon}(t)=a(t)+g_{\epsilon}\left(t, x_{\epsilon}(t)\right) \int_{0}^{1} k(t, s) f_{\epsilon}\left(s, x_{\epsilon}(s)\right) d s \\
=a(t)+\left(g\left(t, x_{\epsilon}(t)\right)+\epsilon\right) \int_{0}^{1} k(t, s)\left(f\left(s, x_{\epsilon}(s)\right)+\epsilon\right) d s \\
>a(t)+g\left(t, x_{\epsilon}(t)\right) \int_{0}^{1} k(t, s) f\left(s, x_{\epsilon}(s)\right) d s .
\end{gathered}
$$

Also

implies

$$
x(t)=a(t)+g(t, x(t)) \int_{0}^{1} k(t, s) f(s, x(s)) d s
$$

$$
x(t)<x_{\epsilon}(t) \text { for } t \in I .
$$

from the uniqueness of the maximal solution (see[13]), it is clear that $x_{\epsilon}(t)$ tends to $q(t)$ uniformly in $t \in I$ as $\epsilon \rightarrow 0$.

By similar way as done above we can prove the existence of the minimal solution.

\section{REFERENCES}

1. J. Banas, K. Goebel, Measure of noncompactness in Banach space, Lecture Note in Pure and Appl. Math. , vol. 60. Dekker, New York, 1980.

2. J. Banas, M. Lecko, W. G. El-Sayed, Eixstence theorems of some quadratic integral equation, J.Math. Anal. Appl. 227 (1998) 276 - 279. 1

3. J. Banas, A. Martinon, Monotonic solutions of a quadratic integral equation of Volterra type, 271 -279, Comput. Math. Appl. 47 (2004).

4. J. Banas, B. Rzepka, Monotonic solutions of a quadratic integral equations of fractional order, J. Math. Anal. Appl. 332 (2007) 1370 -11378.

5. G. Emmanuele, Integrable solution of Hamnmerstien integral equation, 277-284, Applicable Analysis Vol. 50 (1993). 1

6. R. F. Curtain and A. J. Pritchard, Functional Analysis in Modern Applied Mathematics Academic press, 1977.

7. K. Deimling, Nonlinear Functional Analysis, Springer - Verlag, Berlin, 1985.

8. A. M. A. EL-Sayed, H. H. G. Hashem, Carathéodory type theorem for a nonlinear quadratic integral equation, MATH. SCI. RES. J. 12(4),(2008), 71-95.

9. A. M. A. EL-Sayed, H. H. G. Hashem, Integrable and continuous solutions of nonlinear quadratic integral equation, Electronic Journal of Qualitative Theory of Differential Equations 25 (2008), 1-10. 
10. A. M. A. EL-Sayed, H. H. G. Hashem, Monotonic positive solution of nonlinear quadratic Hammerstein and Urysohn functional integral equations, Commentationes Mathematicae, 48, (2008) , 199-207.

11. A. M. A. EL-Sayed, H. H. G. Hashem, Weak maximal and minimal solutions for Hammerstein and Urysohn integral equations in reflexive Banach spaces, Differential Equation and Control Processes, 4 (2008), 50-62.

12. A.M.A. EL-Sayed, H.H.G. Hashem, Monotonic solutions of functional integral and differential equations of fractional order, E. J. Qualitative Theory of Diff. Equ., No. 7. (2009), 1-8.

13. V.Lakshmikantham and S. Leela. Differential and Integral Inequalities, Vol. 1, NewYorkLondon1969.

14. G. Scorza Dragoni, Un teorema sulle funzioni continue rispetto ad una e misurabili rispetto ad un' altra variabile, Rend. Sem. Mat. Univ. Padova 17 (1948), 102-106. 1

${ }^{1}$ Department of Mathematics, Faculty of Science, Alexandria University, AlexanDRIA, EGYPT.

E-mail address: amasayed@hotmail.com

2 Department of Mathematics, Faculty of Science, Alexandria University, AlexanDRIA, EGYPT.

E-mail address: hendhghashem@yahoo.com 